\title{
Differential vasoactive effects of sildenafil and tadalafil on cerebral arteries -relevant to migraine?
}

\author{
Christina Kruuse ${ }^{1,2,3^{*}}$, Saurabh Gupta ${ }^{3}$, Elisabeth Nilsson ${ }^{4}$, Lars Kruse ${ }^{3}$, Lars Edvinsson ${ }^{1,4}$ \\ From 5th International Conference on cGMP: Generators, Effectors and Therapeutic Implications \\ Halle, Germany. 24-26 June 2011
}

\section{Background}

Phosphodiesterase 5 (PDE5) is associated with migraine pathophysiology, stroke recovery and vasospasm treatment $[1,2]$. We have shown previously that vasodilatation was not a prerequisite for migraine induction; sildenafil elicited migraine-like attacks in migraine patients without measurable changes in intra- or extracerebral artery diameter. Further, sildenafil was found to not affect neurovascular response or excitability [3]. However, dural artery responses were not accounted for in the human studies and minor vascular changes of functional importance may not have been detected.

The potential vascular interplay of PDE5 inhibitors sildenafil, tadalafil and UK-114,542 were studied by intraversus extra-luminal administration in rat middle cerebral arteries (MCA) in vitro and on middle meningeal arteries (MMA) in vivo.

\section{Aim \\ To examine a possible vascular site of action, if any, of each of sildenafil and tadalafil by investigating 1 ) the effects of PDE5 inhibitors in vitro dilatation of the mid- dle cerebral artery (MCA) with controlled luminal or extra-luminal application of the drugs and 2) the in vivo effects of intravenous PDE5 inhibitors on the middle meningeal artery (MMA) dilatation in a closed cranial window model in rats.}

\section{Methods}

Rat MCA diameter was investigated using pressurised arteriography, applying UK-114,542, sildenafil, and

\footnotetext{
* Correspondence: ckruuse@dadlnet.dk

'Department of Clinical Experimental Research, Glostrup Hospital, Univ.

Copenhagen, Denmark

Full list of author information is available at the end of the article
}

tadalafil intra- or extra-luminally. Effects on MMA were studied in the in vivo closed cranial window model.

\section{Results}

At high concentrations, abluminal sildenafil and UK114,542 , but not tadalafil, induced dilatation. Luminal application elicited a contraction of $4 \%$ (sildenafil, $\mathrm{p}=$ 0.03 ) and $10 \%$ (tadalafil, $\mathrm{p}=0.02$ ). In vivo, sildenafil, but not tadalafil, dose-dependently dilated MMA concomitant to blood pressure reduction (1-3 mg/kg);1 mg/kg sildenafil inducing $60 \pm 14 \%(\mathrm{p}=0.04)$ and vehicle (DMSO) $13 \pm 6 \%$ dilatation.

\section{Conclusion}

PDE5 inhibitors applied luminally had contractile effect on MCA. Abluminal sildenafil induced MCA dilatation above therapeutic levels. In vivo, sildenafil dilated MMA. Tadalafil had no dilatory effects. PDE5 inhibitors show differential vascular activity in arteries, although clinically the potential for headache induction appears similar. Such findings support clinical studies showing no vasodilatory effects of sildenafil on cerebral arteries in healthy subjects.

\section{Acknowledgements}

The study was supported by Lundbeck Foundation Centre for Neurovascular signalling (LUCENS), Cool Sorption Foundation; CK was supported by grant from the Danish Medical Research Council.

\section{Author details \\ 'Department of Clinical Experimental Research, Glostrup Hospital, Univ. Copenhagen, Denmark. ${ }^{2}$ Department of Neurology, Glostrup Hospital, Univ. Copenhagen, Denmark. 'undbeck Foundation Center for Neurovascular Signalling (LUCENS), Glostrup Hospital, Univ. Copenhagen, Denmark. ${ }^{4}$ Division of Experimental Vascular Research, Department of Internal Medicine, University Hospital of Lund, Sweden.}

Published: 1 August 2011 


\section{References}

1. Kruuse C, Thomsen LL, Birk S, Olesen J: Migraine can be induced by sildenafil without changes in middle cerebral artery diameter. Brain 2003, 126:241-247.

2. Zhang R, Wang Y, Zhang L, Zhang Z, Tsang W, Lu M, et al: Sildenafil (Viagra) induces neurogenesis and promotes functional recovery after stroke in rats. Stroke 2002, 33:2675-2680.

3. Kruuse C, Hansen AE, Larsson HB, Lauritzen M, Rostrup E: Cerebral haemodynamic response or excitability is not affected by sildenafil. $J$ Cereb Blood Flow Metab 2009, 29:830-839.

doi:10.1186/1471-2210-11-S1-P42

Cite this article as: Kruuse et al: Differential vasoactive effects of sildenafil and tadalafil on cerebral arteries -relevant to migraine? BMC Pharmacology 2011 11(Suppl 1):P42.

Submit your next manuscript to BioMed Central and take full advantage of:

- Convenient online submission

- Thorough peer review

- No space constraints or color figure charges

- Immediate publication on acceptance

- Inclusion in PubMed, CAS, Scopus and Google Scholar

- Research which is freely available for redistribution

Submit your manuscript at www.biomedcentral.com/submit 